\title{
La presse écrite togolaise, acteur et témoin de l'ère Eyadéma (1967-2005)
}

The Togolese Press: A Key Political Player in and Witness of the Eyadema Era (1967-2005)

\section{Brice Rambaud}

\section{QpenEdition}

\section{Journals}

Édition électronique

URL : http://journals.openedition.org/transcontinentales/415

ISBN : 978-2-8218-1408-0

ISSN : $1775-397 X$

Éditeur

Editions de la maison des sciences de l'homme

\section{Édition imprimée}

Date de publication : 30 juin 2006

Pagination : $57-76$

ISBN : 2200-92169-1

ISSN : 1950-1684

\section{Référence électronique}

Brice Rambaud, «La presse écrite togolaise, acteur et témoin de l'ère Eyadéma (1967-2005) »,

Transcontinentales [En ligne], 2 | 2006, document 5, mis en ligne le 30 septembre 2012, consulté le 07 septembre 2020. URL : http://journals.openedition.org/transcontinentales/415

Ce document a été généré automatiquement le 7 septembre 2020

Tous droits réservés 


\title{
La presse écrite togolaise, acteur et témoin de l'ère Eyadéma (1967-2005)
}

The Togolese Press: A Key Political Player in and Witness of the Eyadema Era (1967-2005)

\author{
Brice Rambaud
}

1 Depuis un an, au Togo, l'heure est au bilan. Le bilan de l'ère Gnassingbè Eyadéma, qui a régné sans partage sur le pays pendant trente-huit ans, depuis sa prise de pouvoir en 1967 jusqu'à sa mort, en février 2005. Derrière cette apparente stabilité politique, la crise économique s'est installée et a fait trembler le régime au début des années 1990, avec le mouvement populaire qui a débouché sur la reconnaissance du multipartisme et la libéralisation des médias. La suite est connue : Eyadéma s'est maintenu par la force la répression s'accentuant aussi dangereusement que la prédation des ressources de l'État - et par l'organisation d'élections contestables.

2 Au niveau médiatique, après trois décennies de contrôle étatique de l'information et de culte de la personnalité, la libéralisation a permis la création d'une presse privée engagée, dénonciatrice mais très artisanale. Acteur politique à part entière, la presse écrite constitue aussi un bon reflet de la situation sociopolitique du pays. D'une part, elle fait exister la liberté d'expression et le pluralisme politique, met en scène l'idéologie du régime, publie les chuchotements de la rue et rend publiques les divisions de l'opposition. D'autre part, elle éclaire et vit au quotidien une situation socio-économique défavorable, un État qui a gardé ses réflexes autoritaires et elle médiatise le vocabulaire politique innovant de la rue, pour qui politisation et désillusion riment avec dérision. Enfin, la présence des structures et représentations de l'oralité dans le texte journalistique met bien en lumière l'hybridation des logiques importées, héritées de la colonisation, et des répertoires culturels locaux - instrumentalisés ou non. Hybridation perceptible dans toutes les strates du politique et du social. 
3 L'interdépendance et les similitudes entre les champs politique et médiatique sont frappantes au Togo. Grâce à ce phénomène de mimétisme, un autre bilan peut être dressé : celui de la presse écrite sous Eyadéma, qui permet d'analyser les dynamiques politiques internes et externes d'un État africain contemporain. Après un rapide historique de ce medium au Togo, puis un état des lieux en 2003, nous étudierons son contenu, en dégageant ses deux rhétoriques dominantes - celle du pouvoir qui instrumentalise un imaginaire local, et celle des gouvernés, empreinte de dérision. Les trajectoires de réinterprétation de l'oralité qui se sont mises en place dans l'écrit journalistique nous retiendront ensuite.

\section{L'importance originelle des « dynamiques du dehors »}

4 Pratique occidentale, la presse écrite arrive au Togo à la fin du XIX`siècle. Adoptée par les premiers Africains instruits dans l'entre-deux-guerres, son développement reste tout au long du XXe siècle soumis à des influences étrangères qui viennent de l'ancienne métropole et/ou sont liées au contexte politique du continent africain.

\section{La presse au Togo : une pratique héritée de la colonisation}

Les missions chrétiennes allemandes créent les premières publications au Togo, à la fin du XIX siècle ${ }^{1}$. Indépendants, les missionnaires prennent position contre l'escla-vage et la violence $\mathrm{du}$ colonialisme dans quelques feuilles à très faible diffusion. L'administration coloniale allemande, qui a officiellement conquis le territoire en 1884 ${ }^{2}$, publie ensuite à partir de 1906 son journal officiel, Amsblatt.

6 La signature du traité de Versailles le 28 juin 1918 oblige l'Allemagne à renoncer à ses colonies. Le Togo est placé sous mandat de la Société des Nations (SDN) jusqu'en 1945, mandat confié à la France et à la Grande-Bretagne qui tracent en 1919 une nouvelle ligne de partage du Togo : les Français reçoivent 61 \% de l'ancien Togo allemand; les Anglais intègrent leur acquisition territoriale à la Gold Coast, actuel Ghana. Le retour des soldats africains de la Grande Guerre et le changement de puissance colonisatrice entraînent un relatif développement de la presse au Togo et au Dahomey voisin. Des journaux sont communs aux deux pays, comme L'Éveil du Togo, lancé en 1931, qui devient en 1933 L'Éveil du Togo-Dahomey. Ces publications favorisent la circulation d'idées des Noirs « évolués » selon la terminologie coloniale et des Européens.

7 En 1945, le Togo est placé sous tutelle de l'ONU, dont l'administration est confiée à la France. Il acquiert le statut particulier de «territoire associé », membre de l'Union française, et une vie politique active y démarre très tôt. Dès la fin des années 1940, un multipartisme effectif s'installe : une dizaine de formations politiques voient le jour et s'affrontent lors de plusieurs consultations électorales tandis que de nombreux journaux sont créés. Très partisane car directement liée aux organisations politiques, la presse togolaise est alors souvent polémique. Elle relaie les rivalités entre les différents leaders et les légitime ainsi comme interlocuteurs auprès des dirigeants français. Lieu d'expression du politique, la presse joue un rôle important dans la formation de l'élite politique et dans la diffusion de ses idées.

8 Deux organes politiques s'affrontent à cette époque: le Comité de l'union togolaise (CUT) de Sylvanus Olympio, anticolonialiste et partisan d'une indépendance immédiate, 
et le Parti togolais du progrès (PTP) de Nicolas Grunitzky, francophile et favorable à une émancipation progressive. Les nationalistes, proches du CUT, lancent des journaux imprimés en zone anglaise, comme The Ewe News Letter, et des publications en français, L'Unité togolaise (1956-1959) par exemple. Le PTP dispose aussi de publications pour le débat politique: La Flèche et $L a$ Voix du centre (créés en 1955). Parallèlement à ce développement assez pluraliste de l'écrit, les syndicats publient entre 1948 et 1955 Travailleur togolais. Une presse des Européens persiste : l'administration coloniale fait paraître Togo-Français, puis, après 1955, Togo-Républicain.

Si le PTP remporte les élections de 1956 relatives à l'autonomie interne par un jeu d'alliances, c'est le CUT qui gagne les consultations de 1958 relatives à l'octroi de l'indépendance. Sylvanus Olympio, son leader, devient Premier ministre. Deux ans plus tard, le 27 avril 1960, le Togo accède officiellement à l'indépendance.

La période correspondant à la fin de la colonisation se caractérise par un libéralisme politique indéniable qui se traduit par l'introduction d'institutions représentatives héritées du colonisateur, de multiples partis politiques et organes syndicaux, d'une presse assez libre. Cette vie politique n'est cependant pas "démocratique »: elle reste l'apanage des gens instruits, qui ont fréquenté l'école coloniale. La presse constitue un produit et un vecteur d'acculturation ${ }^{3}$ majeur car elle représente l'une des premières formes de l'introduction de l'écrit en Afrique. Instrument du débat politique, elle change de statut après l'indépendance. L'imposition du parti unique sonne le glas d'une presse pluraliste et favorise un journalisme au service du politique.

\section{D’Olympio à Eyadéma : monopartisme et monopole médiatique dans le Togo indépendant}

11 Le 9 avril 1961, Sylvanus Olympio est élu premier président de la République du Togo. Il met en place un régime présidentiel fondé sur un fort pouvoir personnel qui recourt aux détentions arbitraires, gère l'économie de façon équilibrée et désire s'affranchir totalement de l'influence de la France. Olympio impose en 1961 le parti unique. À la fin de cette même année, le CUT devient le Parti de l'unité togolaise (PUT) et proclame Olympio président à vie du Togo.

Dans le domaine des médias, une nouvelle loi limite les critiques de la presse. Avec l'avènement $d u$ parti unique, le pluralisme de la presse s'enrhume: il ne reste plus qu'une dizaine de titres au lendemain de l'indépendance. Les Échos du Togo, Notre Combat ainsi que d'autres journaux du PTP disparaissent progressivement. En 1962, l'État lance Togo-Presse, d'abord hebdomadaire tirant à environ 8000 exemplaires, puis quotidien. Comme dans toute l'Afrique francophone, l'indépendance est marquée par la restriction de la liberté de presse et le quasi-monopole des médias gouvernementaux au nom de «l'union nationale ».

Olympio est tué lors du coup d'État conduit le 13 janvier 1963 par des militaires togolais démobilisés de l'armée française et mis à l'écart depuis leur retour au pays. L'auteur de l'assassinat est le sergent Étienne Eyadéma ${ }^{4}$, alors inconnu en son pays. Promu chef d'État major des armées, Eyadéma prend véritablement le pouvoir en 1967, appuyé par la cellule africaine de l'Elysée ${ }^{5}$. Sa politique de "réconciliation nationale » passe par l'interdiction des partis politiques en 1967 et la création du parti unique, le Rassemblement du peuple togolais (RPT), en 1969. Investi des pleins pouvoirs, il fait du RPT un instrument de répression et de glorification de sa personne tandis que se 
développent progressivement corruption et népotisme au sommet d'un État-parti prédateur, monopolisé par des Kabyés ${ }^{6}$. Les années 1970 et 1980 sont aussi celles des détentions arbitraires et des assassinats politiques. Le régime est de plus en plus impopulaire.

Le pouvoir personnel d'Eyadéma s'appuie sur la présence quotidienne des militaires dans le pays et sur un monopole des médias qui font de lui le «Guide la nation ». En 1974, Eyadéma troque «Étienne » contre un prénom traditionnel, Gnassingbé, dans le cadre de la mise en œuvre d'une politique culturelle d'«authen-ticité » calquée sur celle du Zaïrois Mobutu 7 . Une mythologie officielle du régime apparaît : Eyadéma serait né pour le pouvoir et protégé par le divin. Les mondes artistiques et traditionnels sont mobilisés pour la propagande du chef, dans le cadre d'une stratégie "d'animation politique » et de déification d'Eyadéma ${ }^{8}$. Les médias d'État, Radio Lomé (créée en 1953), Togo-Presse, et la Télévision togolaise (TVT) lancée en 1973, sont au service d'une cause unique : la propagande du RPT et de son chef. Le quotidien Togo-Presse consacre sa Une presque systématiquement au Président. Communiqués, déclarations officielles, culte de la personnalité composent le contenu du seul journal togolais. La critique est interdite.

La «poigne de fer despotique ${ }^{9}$ " du régime se durcit toujours un peu plus. Ce n'est qu'au début des années 1990 que la population togolaise, encouragée par un contexte international propice au changement, se lève contre l'autoritarisme.

\section{Entre facteurs internes et externes : la libéralisation des médias}

L'affaiblissement de l'URSS à la fin des années 1980 a des conséquences directes sur les États africains alignés sur le modèle soviétique. Au Bénin, le marxiste-léniniste Kérékou est contesté par le peuple à la fin de l'année 1989. Une « conférence nationale des forces actives » en février 1990 conduit à la libéralisation de la presse et à la tenue d'élections multipartites libres en mars, remportées par Nicéphore Soglo. Cette alternance politique par voie légale donne des idées au reste du continent.

Le "vent de l'est" se propage au Togo voisin ${ }^{10}$ où le processus de transition démocratique prend un caractère très violent à cause de l'intransigeance du pouvoir en place. Plusieurs facteurs sont à l'origine de ce mouvement de contestation. Facteurs endogènes: l'usure d'un pouvoir corrompu, la crise économique, l'augmentation des inégalités ainsi que la langue de bois des médias d'État et la confiscation du droit d'expression de la population entraînent les revendications démocratiques et la faillite des médias gouvernementaux. Facteurs exogènes: le nombre croissant d'étudiants africains dans les pays occidentaux, la réception des médias internationaux ${ }^{11}$ - qui rendent compte de l'alternance des partis au pouvoir en Europe de l'Est et rendent illusoire la censure des gouvernements africains - contribuent au désir de changement tandis que le président François Mitterrand, dans le discours de La Baule prononcé par lors du XVI Sommet franco-africain, en juin 1990, conditionne l'aide publique au développement de la France à la démocratisation des régimes.

18 Au Togo, dès 1990, des feuilles à faible diffusion comme Le Courrier du Golfe ou ForumHebdo critiquent durement le pouvoir. Les premières manifestations, fin 1990 et début 1991, sont violemment réprimées par les Forces armées togolaises. Les exécutions extrajudiciaires se multiplient. Les affrontements entre population et forces de l'ordre en avril-mai 1991, puis la grève générale du 6 juin 1991 font céder Eyadéma : il accepte la 
tenue d'une conférence nationale qui commence le 8 juillet. Tous les partis et les groupements de citoyens se réunissent autour d'une table pendant cinquante-deux jours. Les retransmissions quotidiennes à la télévision et à la radio captivent la population. Les opposants peuvent enfin parler. Les langues se délient. Les brutalités exercées par le pouvoir pendant les vingt dernières années sont révélées à la population, souvent médusée. La Conférence se transforme en catharsis nationale. La transition démocratique demeure cependant dans l'impasse. Entre coups de force sanglants ${ }^{12}$, divisions de l'opposition, adresse politique et soutien de la France, Eyadéma réussit à se maintenir au pouvoir. Il remporte les élections présidentielles de 1993, 1998 et 2003, qui sont chaque fois une triste démonstration de force militaire, de corruption et d'irrégularités ${ }^{13}$.

Si la transition démocratique demeure dans l'impasse, deux revendications démocratiques ont abouti au début des années 1990: le multipartisme et la libéralisation des médias. La première réforme engendre de nouveaux partis, de nouvelles institutions, l'organisation d'élections et le développement d'associations de la société civile, mais l'absence d'alternance et l'inexistence de la séparation des pouvoirs limitent rapidement ce nouveau multipartisme. La seconde réforme apparaît comme un geste de bonne volonté - en direction du peuple et de la communauté internationale - peu dangereux pour le pouvoir à cause du faible taux de pénétration de la presse ${ }^{14}$, sur lequel on reviendra.

Cette libéralisation des médias entraîne une floraison de journaux privés. Après des décennies de contrôle de l'information, la presse renaît. C'est le "Printemps de la presse ", commun à de nombreux pays d'Afrique francophone. Des dizaines d'hebdomadaires et d'« irrégulomadaires » sont créés par des intellectuels, des avocats, des professeurs, etc. Très virulents à l'égard du pouvoir, ils rendent compte de l'évolution de la situation politique. La "nouvelle presse ", indépendante du pouvoir mais très proche des nouveaux partis politiques, est une presse d'opinion, de dénonciation, de revendication de justice sociale, clairement engagée contre le RPT. Le journaliste redevient un témoin politique et un « acteur incontournable de la vague de démocratisation des régimes politiques ${ }^{15}$ ". À côté de cette presse d'opposition, des titres sont lancés par le parti au pouvoir pour mieux polémiquer et contrer la critique. Ces journaux privés « de la mouvance présidentielle » reprennent la ligne éditoriale du quotidien Togo-Presse: intérêt exclusif pour le RPT, condamnation des partis d'opposition et exaltation du sentiment d'union autour d'Eyadéma.

Durant toute la décennie 1990, le champ journalistique montre un dynamisme remarquable. La profession se structure progressivement mais subit de fortes contraintes politiques et sociales.

\section{Précarité et manichéisme d'une presse de fin de règne}

L'analyse de la presse togolaise présentée ici - structures, contenus, réception s'appuie sur une étude de terrain réalisée entre juin et août 2003 ainsi que sur un corpus de 100 journaux constitué durant ces trois mois. Nous avons choisi cette période car elle correspond au lendemain de la troisième élection présidentielle du Togo dit «démocratique », qui a eu lieu le 1a juin 2003, c'est-à-dire au dernier temps fort du règne d'Eyadéma. Il s'agit d'un moment clé de performance du politique, miroir grossissant des antagonismes. 
La presse togolaise compte 45 titres en 2003. Cette apparente diversité cache une homogénéité certaine: il s'agit d'une presse d'opinion, encore très liée aux partis politiques et dont le développement se heurte à de fortes contraintes locales. La centaine de journaux étudiés correspond à une quinzaine de titres, qui se répartissent entre la presse étatique ( 40 numéros) et la presse privée (60 numéros pour 15 titres environ) pro- ou anti-gouvernementale (respectivement 25 et 35 numéros). Le tableau ci-dessous dresse une typologie de ce corpus.

Tableau 1

\begin{tabular}{|c|c|c|c|}
\hline Catégorie. & Nom & Date de création & Directeur de publication \\
\hline $\begin{array}{l}\text { Presse etatique } \\
\text { (quotidienne) }\end{array}$ & logo-Presse & 1902 & Remy Banafey Assih \\
\hline $\begin{array}{l}\text { Presse privée } \\
\text { de la mouvance } \\
\text { presidentielle } \\
\text { (hebdomadaire) }\end{array}$ & $\begin{array}{l}\text { Echos d Afrique } \\
\text { le Cloiron } \\
\text { Le Destin } \\
\text { Le Gurde } \\
\text { Politicos } \\
\text { Inngo Tingo }\end{array}$ & $\begin{array}{l}2001 \\
2002 \\
2003 \\
\text { inconnue } \\
\text { inconnue } \\
1998 .\end{array}$ & $\begin{array}{l}\text { Abdou Bawa } \\
\text { John Magnétine } \\
\text { Yaspis Pfapfu } \\
\text { Aicha Koumai } \\
\text { Germain Poull } \\
\text { Augustin Assiobo }\end{array}$ \\
\hline $\begin{array}{l}\text { Presse privee } \\
\text { d'opposition } \\
\text { (hebodonadaire) }\end{array}$ & $\begin{array}{l}\text { Carrefour } \\
\text { Crocodile } \\
\text { La Iribune du peuple } \\
\text { La Depeeche lo } \\
\text { Le Combat du peuple } \\
\text { Le Regard } \\
\text { Le Reporter des temps } \\
\text { noureaux } \\
\text { LEvenement } \\
\text { Uotion dinformation }\end{array}$ & $\begin{array}{l}1991 \\
1993 \\
2002 \\
1993 \\
1094 \\
1096 \\
109 \\
1998 \\
1999 \\
1092\end{array}$ & $\begin{array}{l}\text { Holonou Hounkpati } \\
\text { Francis Pedro Amuzun } \\
\text { Kodjo Atantchoo Siliodin } \\
\text { Apollinaire Mewenemesse } \\
\text { Lucien Messan } \\
\text { Abass Derman } \\
\text { Augustin Amega } \\
\text { Philppe Evegnon } \\
\text { Jean-Plerre Tettegan }\end{array}$ \\
\hline
\end{tabular}

Typologie des journaux étudiés (2003)

\section{Une presse d'opinion divisée}

Les acteurs de la presse togolaise répartissent celle-ci en trois grandes catégories : la presse étatique, la presse de la mouvance présidentielle et la presse privée d'opposition.

Togo-Presse, journal d'État, est toujours le seul quotidien du pays. Disposant de seize pages, il publie cinq numéros par semaine, avec une déclinaison du week-end qui porte le nom de Togo-Presse magazine. Vendu 100 francs CFA - ce qui représente une somme non négligeable dans un pays où un instituteur gagne 21000 francs CFA et où le Produit national brut par habitant était en 2002 de 270 dollars américains (soit 180000 francs $\mathrm{CFA}$ ) -, il tire à 5000 exemplaires. Togo-Presse est distribué très majoritairement à Lomé, où $80 \%$ de ses ventes sont réalisées, mais aussi dans les grandes villes du pays (notamment à Kara, deuxième ville du Togo et fief du pouvoir). La vente s'effectue à la criée (aux carrefours des grandes routes), dans des dépôts et surtout par abonnement: legs du parti unique, la quasi-totalité des administrations publiques et entreprises para-étatiques est abonnée à Togo-Presse, organe d'État. Son contenu évitant toute critique envers le pouvoir, Togo-Presse est le journal des informations officielles, des communiqués des différents ministères, des appels d'offres, des annonces 
nécrologiques. On note la présence d'informations traduites en langues nationales éwé et kabyé mais aussi en anglais pour satisfaire les Ghanéens présents au Togo. Malgré son rôle gouvernemental, Togo-Presse dispose de moyens de fonctionnement des plus limités, ce qui explique les salaires souvent payés en retard, la qualité moyenne de l'impression, la lenteur de son informatisation.

Toutes les autres publications sont privées et périodiques. Journaux d'opposition ou de la mouvance présidentielle, ces hebdomadaires et mensuels sont extrêmement politisés dans leur contenu et dans leur ligne éditoriale. La dénonciation politique, la proximité des directeurs de publication avec les partis ainsi que la difficulté d'accéder à l'information officielle en font une presse d'opinion dans laquelle faits et commentaires sont totalement imbriqués.

Les hebdomadaires privés d'opposition sont vendus 250 francs CFA. Ce sont tous des journaux de huit pages. La production et la diffusion restent très majoritairement centralisées à Lomé, capitale du Togo, même si certains titres sont distribués dans les autres grandes villes: seulement $15 \%$ de la production est diffusée dans quelques préfectures du pays ${ }^{16}$. Les journaux peinent à pénétrer la campagne à cause de la médiocrité des circuits de distribution et des infrastructures routières. Le Regard est le plus lu, avec 3500 exemplaires par semaine. Les autres journaux d'opposition - comme Le Reporter des temps nouveaux, Carrefour, Le Scorpion Akeklé, Crocodile - diffusent entre 1500 et 2500 exemplaires. Les tirages sont donc très faibles.

28 La presse privée de la mouvance présidentielle, proche du pouvoir, est encore moins lue: Politicos, Échos d'Afrique, Le Clairon, Le Destin impriment entre 500 et 1000 exemplaires par semaine. Cette presse s'en sort cependant mieux que la presse d'opposition car son financement provient fréquemment d'hommes forts du pouvoir ou de partis satellites du RPT.

29 Trois grandes catégories donc, mais deux types de discours seulement, car le discours de la presse de la mouvance présidentielle rejoint pleinement celui de Togo-Presse dans le soutien sans faille au régime. Cet unilatéralisme trouve sa parfaite contrepartie dans le discours de la presse d'opposition, qui rejette massivement le pouvoir RPT. Il y a bien ici deux visions antagonistes d'Eyadéma, du régime, du fait politique, du journalisme, des aspirations du peuple ${ }^{17}$ - phénomène qui trouve sa source dans le manichéisme régnant dans la vie politique togolaise des années 1990-2000. Ajoutons que si les acteurs de la presse au Togo se classent eux-mêmes en deux grands groupes, des divisions existent dans chaque camp : la connivence des journaux avec les hommes politiques qui les financent ou avec celui qu'ils considèrent comme leur "champion" transparait dans leur ligne éditoriale.

Oubliée l'honnêteté journalistique, la presse pro-gouvernementale est au service d'un président populaire, divin, mythique, d'une idéologie à légitimer en employant un discours persuasif. Ainsi, après la dernière élection présidentielle d'Eyadéma en 2003, les irrégularités, la coercition, l'impopularité du pouvoir et la crise économique sont écartées par l'hebdomadaire Le Clairon pour fêter la nouvelle victoire du chef :

«L'Éternel a écouté le peuple qui implore et accepté une fois encore de lui donner

la victoire. [...] La victoire du candidat du peuple Gnassingbé Eyadéma n'est, en

effet, rien d'autre que la réalisation des souhaits des populations togolaises du Nord au Sud et de l'Est à l'Ouest ${ }^{18}$." dictateur, où il est dit « voleur d'élection ${ }^{19}$ » avec un « manque patent de légitimité ${ }^{20}$ ». 
La contestation du chef est partout. Elle se confond avec un discours de moralisation où les journalistes s'assimilent au peuple souverain. La critique envers le pouvoir est très dure ; elle frise fréquemment la diffamation : «Qui a volé, volera ! C'est un dicton qui trouve la plénitude de son sens au pays du RPuTain ${ }^{21}$ " écrit Carrefour à propos des élections et des crimes économiques d'un « régime aux abois qui gouverne le Togo sans gouverner ${ }^{22}$ ».

\section{De fortes contraintes de production}

À cause de ses faibles tirages et du manque crucial de publicité ${ }^{23}$, la presse privée est dans une situation financière instable. Nombreux sont les hebdomadaires éphémères. Il faut dire que les contraintes de production sont fortes et que le lectorat potentiel est limité. En 2001, le taux d'alphabétisation des adultes ne dépassait pas $50 \%$, pour une population très jeune de surcroît ${ }^{24}$ : les $0-14$ ans représentaient cette même année $44,3 \%$ d'une population de 5 millions d'habitants. Avec un indice de développement humain (IDH) classé en 141 position sur 144 pays en 2001 par le PNUD, le Togo ne consacrait que $4,5 \%$ de son produit intérieur brut au budget de l'éducation. Si l'écrit et l'usage de langue française écartent la moitié de la population de la presse, le prix élevé d'un journal freine aussi l'achat. La crise économique due à la chute du cours des matières premières dans les années 1980 et à l'arrêt de la coopération internationale après 1993 - exception faite de la coopération française - avec l'État togolais touchent aussi pleinement la presse.

La presse reste un fait urbain. C'est dans les villes que se trouve son public : les lettrés, intéressés par la chose publique, disposant de moyens financiers suffisants, et les fonctionnaires dont les administrations sont abonnées à certains journaux. Le public de la presse est cependant élargi par la lecture collective (un lettré lit au groupe) et le fort taux de circulation des journaux (un journal acheté passe entre des dizaines de mains).

Le taux de pénétration de la presse est faible. Le paysage médiatique est dominé par la radio, seul réel mass media en Afrique. Au Togo, la radio est partout. Elle présente les avantages de pouvoir être reçue par les analphabètes à moindre frais, et de couvrir la quasi-totalité du territoire. Apparues, comme la nouvelle presse, à la faveur du mouvement de démocratisation, les radios privées se sont aussi multipliées : on en dénombrait plus de 70 en $2004{ }^{25}$. Un autre medium constitue un moyen d'information majeur: Radio-Trottoir, ou la palabre comme mode de transmission des nouvelles. La discussion reste le «lieu» symbolique du lien social et du rapport à l'Autre indispensable à la vie économique, culturelle, intellectuelle, sociale de l'individu et du groupe. Radio-Trottoir prolonge et, souvent, déforme les informations entendues à la radio. La parole est aussi soustraite à la pression des autorités ${ }^{26}$.

Enfin, les contraintes politiques sont fortes. Elles interviennent pleinement dans la chaîne de production de la presse : saisies de journaux avant publication, pressions téléphoniques, incarcérations abusives, exils de journalistes se sont répétés dans les années 1990, illustrant l'autoritarisme d'un pouvoir critiqué ${ }^{27}$. Avec une liberté de la presse malmenée, l'autocensure fonctionne à plein tandis que les enquêtes sont difficilement réalisables. 


\section{Un rôle politique fort}

éditoriales des différents titres de la presse privée d'opposition. Ainsi Motion d'information est un hebdomadaire proche du parti Union des démocrates pour la solidarité (UDS-Togo), Le Reporter soutient le parti de la Convergence patriotique panafricaine (CPP), etc. Le financement de certains journaux par des partis et l'influence sur la ligne éditoriale de la sensibilité politique du directeur de publication - qui cumule fréquemment les fonctions de directeur, rédacteur en chef, éditorialiste et gestionnaire - expliquent que la presse d'opposition épouse les lignes de divisions de l'opposition. Cette proximité des journaux avec les politiques n'empêche cependant pas, parfois, qu'un journaliste critique le parti qui le finance.

40

Censé permettre la prise de parole et retranscrire les aspirations de la population, notamment à des travers les éditoriaux, l'écrit journalistique possède une fonction critique importante, qui a des conséquences directes sur la gouvernance : «En sus des pressions, voire des interventions extérieures en faveur de la liberté de la presse, la 
pression interne est, bien souvent, non négligeable. Les pouvoirs en place sont de plus en plus sensibles aux critiques venues du dedans. ${ }^{30}$ »

41 La presse tire sa force politique de la position sociale de son lectorat. Journalistes et public de la presse sont issus du même milieu social: au Togo comme dans toute l'Afrique francophone, «la presse privée est un message de l'élite pour l'élite: la communication se joue dans la petite communauté restreinte de ceux qui, grâce à leur position sociale et culturelle, peuvent se permettre d'approcher la chose politique ${ }^{31}$ ». La presse informe l'élite décisionnelle. Elle s'adresse à des leaders d'opinion, très influents dans leur(s) communauté(s) d'appartenance, qui vont relayer l'information ou l'opinion du journal. Medium des divisions politiques, la presse est aussi celui de la « reliance sociale ${ }^{32}$ ", de l'assimilation réciproque d'une élite en formation ${ }^{33}$.

La presse a une fonction cathartique : les prises de positions virulentes, brutales des journalistes contre le camp politique adverse et l'importance du courrier des lecteurs (une page sur huit en moyenne dans la presse d'opposition), souvent acerbe contre le régime, ont aussi pour but de satisfaire le besoin de dire son indignation et de canaliser les rancœurs des journalistes et du public.

43 Le pouvoir politique de la presse togolaise séduit les bailleurs de fonds depuis les années 1990. Il est l'objet de toutes les attentions des organisations démocratiques internationales et de la coopération française, qui désirent renforcer le rôle du journaliste dans la démocratisation et dans la construction de l'État-nation. De nombreuses formations ponctuelles à l'écriture journalistique, à la déontologie, au fonctionnement d'une entreprise de presse sont proposées aux journalistes togolais, principalement par le service de coopération et d'action culturelle de l'ambassade de France et par le service formation de Radio France Internationale. Elles répondent à un réel besoin car il n'y a pas d'école de journalisme au Togo. Mais elles constituent également un retour puissant de l'influence française sur le journalisme togolais, puisque ces formations dispensent du savoir-faire français, avec des formateurs français, et imposent les normes du modèle français de journalisme ${ }^{34}$ à des journalistes africains qui ont déjà développé des manières de faire locales: le traitement du fait politique et l'écriture de presse ont en effet une tonalité typique au Togo.

\section{La création d'un journalisme local : de la dérision à I'hybridation}

L'analyse de l'écriture journalistique met en lumière deux rhétoriques opposées : l'une de la contestation, qui utilise un langage de dérision révélant la désillusion de la rue face à une transition démocratique au point mort; l'autre du pouvoir, qui instrumentalise un imaginaire local par des techniques de propagande universelles.

\section{Images et dérision}

45 Figures de style privilégiées, les images verbales sont omniprésentes de le texte. Tout article est rendu vivant par le recours à des métaphores, à des comparaisons qui interpellent le lecteur, et qui font appel à ses références culturelles. La presse d'opposition use beaucoup d'expressions imagées pour dénoncer le pouvoir : ainsi Le Reporter perçoit le régime Eyadéma comme « une dictature sans visage », « aux abois » 
et considère que ses dignitaires ont "une furie épidermique du pouvoir» ${ }^{35}$. Cette même presse d'opposition aime à filer la métaphore animale à propos du chef de l'État : il faut «arracher le pouvoir des griffes de l'éléphant ${ }^{36}$ " explique l'Événement; «Eyadéma est une sangsue qui vide les Togolais de leur désir, de leur liberté, de leur personnalité ${ }^{37}$ » accuse la Tribune du peuple. Le président est également surnommé « le Timonier », « le boss » ou le « baobab». Ce recours systématique à une écriture imagée, censée dynamiser le texte, dénote l'absence de formation de la grande majorité des journalistes, qui écrivent comme ils parlent et désirent toucher le lecteur en utilisant le même langage que lui. On est bien dans la théâtralisation du politique : les figures de style permettent de simplifier le discours; le journaliste essaie d'étonner le lecteur et fait appel à l'imagination pour faire passer des émotions, pour susciter l'empathie. L'image verbale concrétise alors avec force une forme inconsciente de persuasion. Elle permet de désacraliser l'autorité du chef, de dénoncer de façon détournée.

46 À ces images verbales, s'ajoutent des interjections, des adresses directes aux hommes politiques et des allégories ${ }^{38}$. Le vocabulaire des journalistes est également riche en jeux de mots. La presse d'opposition en abuse pour caricaturer la vie politique du pays. L'Événement, appelle Eyadéma "le vieux baobab ", avec une bonne dose d'humour, car un baobab est difficilement déracinable. L'assonance est employée avec récurrence, notamment pour les jeux de mots concernant la figure du chef: pour Tribune du peuple, Eyadéma est "l'unique monarque inique ${ }^{39}$ ». L'ironie est itérative, elle marque une certaine complicité avec le lecteur : «Le peuple a choisi le guide bien aimé le Général Eyadéma ${ }^{40}$ " s'amuse $L a$ Dépêche, qui brocarde ainsi simultanément l'idéologie du régime, les trois élections « démocratiques » remportées par Eyadéma et la presse progouvernementale. Dans le même esprit, L'Événement, journal d'opposition, " félicite » Eyadéma " pour sa brillante réélection » ${ }^{41} \ldots$

47 Métaphores animales, jeux de mots et ironie renvoient à un discours politique marqué par la dérision. Lieu d'expression du politique, la presse reprend les expressions de la rue, les bons mots de Radio-Trottoir. À l'image d'une grande partie de la population pour qui la démocratisation est impossible sans le départ d'Eyadéma, la presse d'opposition érige le rire en mode populaire d'action politique.

Le langage de la dérision politique est ancien au Togo. Pour Comi Toulabor, il constitue même un trait socioculturel des Éwés ${ }^{42}$ qui a survécu à la langue de bois du parti unique et s'est même développé à travers elle : le discours dithyrambique qui a prévalu pendant des décennies a rendu la critique impossible, mais a suscité une verve populaire, clandestine, contestant l'unanimisme imposé. Cette résistance politique du verbe des petits face au discours des forts est sortie de sa clandestinité au début des années 1990, en bénéficiant d'une plus grande liberté d'expression. Elle s'est renforcée à la mesure des espoirs démocratiques déçus.

Marque de défiance face aux régime, la raillerie apporte aussi un brin de légèreté devant une situation politique et économique difficile. Elle est à l'origine d'une grande richesse lexicale, dans les discussions entre amis comme dans la presse écrite: métaphores et surnoms donnés aux hommes politiques, néologismes, glissements de sens, détournements des slogans et des signes officiels sont autant de façons de se moquer du régime. La dérision joue également avec les sonorités: l'Assemblée nationale n'ayant aucun réel pouvoir puisque les décisions importantes sont prises au plus haut niveau de l'exécutif, les députés deviennent dans les bouches et les stylos des « dépipés » ou encore des « députains ${ }^{43}$ » - jeu de mots que l'on peut rapprocher du « 
RPuTain ", utilisé par Carrefour, qui dévie l'acronyme de l'ancien parti unique. La rue a pour sa part consacré à Lomé le RPT comme le Rassemblement des profiteurs togolais !

La presse écrite d'opposition contribue à trouver de bons mots pour tourner le pouvoir en dérision, mais aussi à faire circuler ceux que ses lecteurs - surtout jeunes et urbains - créent chaque jour. Support de la contestation, la presse privée d'opposition médiatise aussi des modes d'action politique provenant du corps social. Elle se différencie alors fondamentalement de la presse pro-gouvernementale, dont l'action se situe plus dans l'instrumentalisation du politique.

\section{Représentations et instrumentalisation}

51 L'étatique Togo-Presse et la presse de la mouvance présidentielle peuvent être considérés comme des instruments au service d'un régime et d'une idéologie: le « langage des puissants » a survécu à la libéralisation. Les journaux recherchent une communication efficace et, pour cela, n'hésitent pas à recourir à des techniques de propagande et à rechercher l'empathie par le recours à un passé mythifié. Leur discours médiatique emploie de nombreux ressorts de la parole manipulée: désinformation et persuasion ; simplification (une idée est exprimée avec force et ne souffre aucune discussion); grossissement (élimination des informations qui pourraient nuire); orchestration (répétition inlassable des idées principales pour les inculquer au public) ; contagion (création d'une illusion d'unanimité).

Ainsi, lors de l'élection de 2003, la presse pro-gouvernementale a insisté sur quatre points pour légitimer un scrutin très contestable ${ }^{44}$ : la transparence du vote, le cautionnement de la communauté internationale, le charisme d'un chef mythique et la victoire du peuple togolais. Pour Le Guide, qui titre «la démocratie togolaise au beau fixe »:

« Dans l'ensemble, le scrutin dernier a été jugé libre, démocratique et transparent. Les observateurs arrivés au Togo dans le cadre de la supervision de ces élections sont unanimement satisfaits $\mathrm{du}$ bon déroulement des opérations et $\mathrm{du}$ dépouillement des bulletins. Il est regrettable que certains Togolais inventent des élucubrations et montages grossiers pour jeter un discrédit sur le bon déroulement des élections passées. ${ }^{45}$ »

53 L'iconographie - photographies et illustrations - joue un rôle important dans la mise en scène des représentations du pouvoir. Elle privilégie les portraits, et particulièrement ceux d'Eyadéma. Le président est partout, aussi bien dans la presse qui lui est inféodée que dans la presse qui le rejette, car il est resté jusqu'à sa mort le point nodal de la vie politique togolaise et de sa médiatisation. Il constitue la raison d'être de la presse togolaise, qu'elle soit apologétique ou critique. Togo-Presse et la presse de la mouvance présidentielle se distinguent en mettant en scène le général-président avec les attributs d'un chef traditionnel fantasmé, notamment le sceptre qui renvoie à l'autorité souveraine et à la maîtrise des forces invisibles, ou l'arrivée à la cérémonie d'investiture en cheval blanc. Un plan large qui représente le chef assis en position d'écoute et de sagesse illustre une position royale. La presse proche du pouvoir met aussi fréquemment en scène les chefs coutumiers reçus par Eyadéma. Ils viennent rappeler la doctrine d'« authenticité culturelle » prônée par le régime, puis légitimer le président par leur notoriété symbolique - ils représentent un pouvoir perpétué - et par la religiosité animiste qu'ils véhiculent: les chefs traditionnels et les forces occultes 
sont avec Eyadéma. La politisation et l'instrumentalisation de la chefferie sont caractéristiques du régime RPT ${ }^{46}$.

$$
\text { trouve confronté à des pratiques et des représentations issues de l'oralité qui prévaut }
$$
dans les différentes cultures togolaises.

\section{La pensée de l'oralité dans l'écrit journalistique}

Les 45 groupes ethniques ${ }^{52}$ du Togo constituent des sociétés fondées sur le collectif et sur l'importance de l'oralité comme mode de transmission de l'information. L'arrivée 
de l'écrit n'a pas bousculé les schèmes de pensée: la nouveauté du medium a été intégrée par les lettrés et l'oralité resurgit au quotidien dans le langage, dans les pratiques et dans les représentations. L'écriture journalistique togolaise recourt à un style oral très prononcé. Il y a bien une hybridation inconsciente de l'écrit et de l'oral, une " pensée de l'oralité dans le texte journalistique ${ }^{53}$ ». L'oral et le rapport au monde qu'il véhicule constituent le socle sur lequel l'écriture d'un journal - mode de communication au départ exogène - vient se greffer et se métisser.

En premier lieu, le discours de la presse togolaise se caractérise par sa qualité performative (« dire, c'est faire »). Sous Eyadéma, la presse d'opposition fait exister la contestation et les partis d'opposition en les rendant visibles dans l'espace public tandis que la presse pro-gouvernementale fait exister l'idéologie du régime. Cette relation se retrouve dans toutes les sociétés orales, où le communicant a une attitude magique à l'égard des mots, «naturellement » performatifs : le verbe donne naissance aux choses ; l'idée et le réel sont intimement imbriqués. Les sociétés de l'oralité sont celles de l'efficacité du verbe. Le langage performatif et la pensée de l'oralité confortent le rapport des journalistes à l'égard des mots, qui tendent à agir sur le monde.

Une deuxième empreinte de l'oralité dans la presse togolaise vient de son fort côté persuasif. Le ton est subjectif et cherche toujours à convaincre le lecteur. Cette efficacité de la parole politique est ancienne. Dans les sociétés togolaises, les détenteurs de l'autorité ne peuvent agir que par la persuasion. C'est la fameuse palabre, au cours de laquelle il faut parfois convaincre tout le monde avant de prendre une décision. Chez les Éwés, « la parole n'est pas un mode passif de communication mais un mode d'action par excellence. La parole se livre comme une arme redoutable et on l'utilise ; il en est ainsi dans les débats tant publics que privés ${ }^{54} »$. Les mots visent l'effet plus que l'information, ils recherchent l'influence durable. Outre l'histoire politique bipolarisée du Togo - symbolisée par la dualité Eyadéma-Gilchrist Olympio ${ }^{55}$-, le manichéisme de la presse togolaise trouve une de ses origines dans la réinterprétation de pratiques politiques anciennes. Opinion et prise de position riment souvent avec simplification pour frapper l'auditoire et convaincre par empathie.

61 Ce manichéisme des acteurs politiques a une conséquence aussi dans le discours des journaux: la catharsis. La palabre et la presse écrite permettent, par l'expression d'opinions aussi antagonistes que violentes, la réduction des conflits. Sous l'arbre à palabre, la parole joue un rôle essentiel dans l'apaisement des tensions en substituant une guerre orale (halo en éwé) à une guerre armée, une joute verbale à une guerre de sang ${ }^{56}$. Elle permet de corriger les inégalités et de préserver la cohésion sociale. La presse reproduit cette pratique: les propos injurieux ou la dérision de la presse d'opposition envers le RPT, et réciproquement les attaques de la presse progouvernementale envers les leaders de l'opposition, permettent un défoulement qui contribue à l'apaisement du conflit politique.

Quant au style, on a dit plus haut comment empreinte de métaphores, de comparaisons, d'ironie, de jeux de mots et de proverbes, l'écriture de la presse togolaise reflète des techniques propres à la narration orale. La pensée de l'oralité est fortement métaphorique. L'usage de la première personne du pluriel, l'ironie, et la présence de nombreux proverbes dans le langage journalistique sont autant d'emprunts à l'oralité, notamment au langage du griot. Le "nous » dont use ce dernier rassemble orateur et auditeur autour d'une cause commune. L'ironie lui permet d'exprimer au Prince les frustrations du groupe. Fréquemment utilisés pour commencer un article, notamment 
un fait divers, et lui donner une valeur universelle, les proverbes représentent encore des points de partage culturels et politiques entre le journaliste et son lecteur. Ils actualisent les héritages culturels et définissent la norme sociale.

Comment ne pas voir en définitive que "dans une société qui reste essentiellement fondée sur la parole, le ton de la presse résonne souvent comme la voix d'un griot ${ }^{57}$ » ? Comment ne pas considérer le journal comme le nouvel arbre à palabre ${ }^{58}$ ? Figure de l'oralité et du groupe, le griot est l'archiviste de la mémoire de la communauté. Il fait partie d'une caste particulière, héréditairement responsable de la transmission du savoir et de l'explication du monde. Narrateur, poète, conteur, il est aussi dénonciateur de certaines frustrations du groupe. On a beaucoup parlé du «journaliste-griot » car, comme le griot, le journaliste en Afrique franco-phone, porte-voix d'un homme politique, est resté la caisse de résonance de son chef. Cependant, si la connivence entre monde politique et monde médiatique s'inscrit dans des héritages socioanthropologiques, elle résulte aussi de paramètres économiques: la précarité financière de la presse la rend plus aisément prompte à chanter les louanges de son financier.

\section{Conclusion}

Le 5 février 2005, le «baobab » est tombé. L'émoi a prévalu dans la presse d'opposition au lendemain de l'annonce du décès d'Eyadéma: pas de satisfaction mais plutôt une peur des lendemains incertains. Elle a vite repris sa fonction critique et sa verve devant une situation politique peu changeante. De leur côté, Togo-Presse et la presse de la mouvance présidentielle pleurèrent des mois durant celui qui était à leurs yeux le Père de la Nation.

Le «Timonier» togolais a été emporté, mais le clan s'est accroché au pouvoir avec l'arrivée du fils préféré à la tête de l'État, Faure Gnassingbé, immédiatement appelé «Faure Vodou » ou « Forevi ${ }^{59}$ " par la presse d'opposition. La transition politique a été houleuse. Le coup d'État constitutionnel puis les élections qui ont suivi le retour à une certaine légalité ont donné lieu à des manifestations et à des affrontements entre forces armées et population. Le retour au calme a été rapide. Faure Gnassingbé, élu, a choisi Edem Kodjo, leader de la CPP, comme Premier ministre. Kodjo est devenu le «Roi» pour toute la presse. Le langage politique n'a rien perdu de son dynamisme.

La presse a peu évolué dans ses structures et dans sa production après le changement de dirigeant. Des journaux ont fermé, d'autres ont été créés, souvent avec les mêmes journalistes. Des espoirs sont nés - lors des négociations avec l'Union européenne pour la reprise de la coopération, l'État togolais a signé plus d'une vingtaine d'engagements démocratiques et le président de la Haute autorité de l'audiovisuel et de la communication (HAAC) est pour la première fois un journaliste issu de la presse d'opposition - mais les problèmes de liberté de presse persistent.

67 Pour autant, l'espace public et la culture politique sont en pleine mutation en Afrique

${ }^{60}$. Les médias reflètent les évolutions en cours mais les impulsent aussi : évolutions sociales, démographiques, politiques; négociation de l'autorité ou actualisation des mythes. Deux rhétoriques symboliques se sont mises en place dans la presse du Togo: celle du pouvoir et celle de la contestation. Si ces rhétoriques sont inversées, elles 
utilisent le même soubassement anthropologique et la même logique politique de compétition.

Medium importé, la presse togolaise s'est vue réappropriée par une société africaine particulière, en fonction de dynamiques socio-anthropologiques qui lui sont propres, des contraintes politiques $\mathrm{du}$ moment, des stratégies personnelles des acteurs. L'hybridation des logiques importées et des répertoires culturels locaux constitue un élément décisif du développement de l'Afrique moderne, de plus en plus pris en compte par la reconnaissance de la pluralité des Afriques. La trajectoire de la presse écrite au Togo navigue constamment entre histoire coloniale, influences extérieures, pratiques universelles et inscription dans des réalités, des contraintes, des façons de faire, des représentations très locales. La forte tension qui anime les sociétés africaines contemporaines, entre le local et le global, entre l'endogène et l'exogène, trouve ainsi dans l'émergence de cette nouvelle réalité médiatique une de ces multiples expressions.

\section{BIBLIOGRAPHIE}

- ADRIAAN E. et B. Van Roueroy Van NieEuWAal, L'État en Afrique face à la chefferie. Le cas du Togo, Paris, Karthala, 2000.

- AgBlEMAGNon F., Sociologie des sociétés orales d'Afrique Noire. Les Eve du Sud-Togo, Paris, Éditions Silex, 1984

- ATTISO F. S., La problématique de l'alternance politique au Togo, Paris, L'Harmattan, 2001.

- BALANDIER G., Le détour. Pouvoir et modernité, Paris, Fayard, 1985.

- BOURGAUlT L., Mass media in Subsaharan Africa, Bloomington IN, Indiana University Press, 1995.

- CORNEVIN R., Histoire du Togo, Paris, Berger-Levrault, 1969 (3 édition revue et augmentée).

- FRÈrE M.-S., Presse et démocratie en Afrique francophone. Les mots et les maux de la transition au Bénin et au Niger, Paris, Karthala, 2000.

- PERRIN A. et J. zoDZI, L'état des médias au Togo, Gret, 2000.

- RAMBAUD B., « Négociation africaine des modèles médiatiques occidentaux. Le cas de la presse togolaise », mémoire de DEA, université Bordeaux 3, 2004.

- TOULABOR C.-M. (dir.), Togo authentique, Politique Africaine, $\mathrm{n}^{\circ}$ 27, mars1987.

- TOULABOR C.-M., Le Togo sous Eyadéma, Paris, Karthala, 1986.

- TUDESQ A.-J., Feuilles d'Afrique, étude de la presse de l'Afrique subsaharienne, Talence, Éditions de la maison des sciences de l'homme d'Aquitaine, 1995.

- TUDESQ A.-J., L'espoir et l'illusion, actions positives et effets pervers des médias en Afrique subsaharienne, Talence, Éditions de la maison des sciences de l'homme d'Aquitaine, 1998. 


\section{NOTES}

1. A.-J. Tudesq, Feuilles d'Afrique. Étude de la presse de l'Afrique subsaharienne, Éditions MSHA, 1995,

p. 23.

2. Le 5 juillet 1884 , le chef Mlapa III de Togoville signe un traité de protectorat avec le représentant de l'Empire allemand, le consul Gustav Nachtigal, faisant du territoire togolais une colonie allemande. Un an plus tard, en 1885, la conférence de Berlin attribue $90400 \mathrm{~km}^{2} \mathrm{au}$ territoire togolais. Le Sud du territoire est conquis, mais les Allemands mettent vingt ans pour occuper tout le pays.

3. Pour une analyse des transferts et des ruptures que représente l'importation des médias en Afrique, voir A.-J. Tudesq, L'espoir et l'illusion. Actions positives et effets pervers des médias en Afrique subsaharienne, Talence, Éditions MSHA, 1998, pp. 13-51.

4. Pour l'historique complet des faits, lire Afrique-Asie $n^{\circ} 33$, juin 1992, pp.11-12. Le jeune militaire Eyadéma revendiquera longtemps cet assassinat, avant de se taire définitivement sur cet acte.

5. F.-X. Verschave, La Françafrique, le plus long scandale de la République, Paris, Stock, 1988, pp. 120-122.

6. Eyadéma étant Kabyé, cette tribalisation de l'État et de l'armée autour de parents (notamment issus du village de Pya) a permis la création d'un pouvoir clanique totalement inféodé au chef de l'État.

7. Eyadéma, à l'image de Mobutu, fonde son régime sur une prétendue "authenticité ", se réclamant de valeurs spécifiquement africaines. Il mobilise la population par des groupes d'animation autour de cérémonies, de chants, de danses pour rejeter les legs de la colonisation et exalter une "africanité » qui se révèle vite instrument puissant de légitimation du pouvoir et d'encadrement du peuple. Lire Angulu M. Edi, Adieu Mobutu, "génie » de Gbadolite: de la dictature authentique à la " démocratie intégrale », Genève, DS Éditions, 1991.

8. C.-M. Toulabor, Le Togo sous Eyadéma, Paris, Karthala, 1986, pp. 105-182.

9. E. Adriaan, B. Van Roueroy Van Nieeuwaal, L'État en Afrique face à la chefferie. Le cas du Togo, Paris, Karthala, 2000, p. 133.

10. L'influence du Bénin limitrophe est non négligeable dans le déclenchement des revendications démocratiques au Togo. Lire R. Banégas, « Retour sur une transition modèle. Les dynamiques du dedans et du dehors de la démocratisation au Bénin », in J.-P. Daloz, P. Quantin (dir.), Transitions démocratiques africaines, Paris, Karthala, 1997, pp. 23-94.

11. Radios internationales et chaînes de télévision par satellite. Lire A. Ba, Télévisions, paraboles et démocratie en Afrique noire, Paris, L'Harmattan, 1996.

12. La spirale de la violence fait des dizaines de victimes dans les années 1992 et 1993. La communauté internationale réagit peu aux atteintes aux droits de l'Homme. Une manifestation pacifique est réprimée dans le sang le 25 janvier 1993 : c'est le «massacre du jardin de Fréau », dont le traumatisme reste vivace (20 morts et une centaine de blessés).

13. Voir les rapports d'Amnesty International publiés après chaque élection, notamment Togo : État de terreur, Amnesty International publications, 1999.

14. En 2002, le taux de pénétration des quotidiens est de 2,2 pour 1000 selon le PNUD.

15. A.-J. Tudesq et $\mathrm{S}$. Nedelec, Journaux et radios en Afrique aux XIX et $X X^{e}$ siècles, Paris, Groupe de recherche et d'échanges technologiques, 1998, p. 150.

16. A.Perrin et J.Zodzi, L'état des médias au Togo, Groupe de recherche et d'échanges technologiques (Gret), 2000. À titre d'exemple, en 2003, Le Reporter des temps nouveaux, deuxième hebdomadaire d'opposition du Togo, met en vente 2500 numéros par édition. Les villes de Dapaong et Aného reçoivent 30 exemplaires chacune tandis que Kpalimé, Atakpamé, Tsévié, Agou et Vogan en reçoivent 20. Autant dire que la couverture du territoire est quasiment nulle en 
dehors de la capitale - sept préfectures se partagent 160 numéros par semaine. Entretien avec A. Améga, directeur de publication du journal Le Reporter, août 2003.

17. Voir l'étude des discours de la presse togolaise lors de l'élection de 2003 dans B. Rambaud, "Négociation africaine des modèles médiatiques occidentaux. Le cas de la presse togolaise ", mémoire de DEA en sciences de l'information et de la communication, université Bordeaux 3, 2004.

18. Le Clairon $\mathrm{n}^{\circ} 24 \mathrm{du} 10$ juin 2003.

19. Motion d'information $\mathrm{n}^{\circ} 229 \mathrm{du} 10$ juin 2003.

20. L'Événement $\mathrm{n}^{\circ} 175 \mathrm{du} 25$ juin 2003.

21. Carrefour $n^{\circ} 363$ du 19 juin 2003.

22. Ibid.

23. Si Togo-Presse a de nombreuses publicités (environ 4 pages sur 16 tous les jours) provenant d'administrations ou d'organismes para-étatiques, la presse privée n'en a quasiment pas, les rares annonceurs ne désirant pas se mettre le régime à dos en publiant dans un journal d'opposition.

24. Voir PNUD, Rapport mondial sur le développement humain, 2003.

25. E. Damome, "Les radios en Afrique et la propagande religieuse : contenus, fonctions et enjeux. Le cas du Togo ", mémoire de DEA, université Bordeaux 3, 2004.

26. Jean-François Bayart voit dans la pratique de Radio-Trottoir un espace pluriel d'interaction et d'énonciation du politique et de l'État, un «murmure » qui traverse les groupes sociaux « et qui déjoue avec insolence les embargos de la censure ». J.-F. Bayart, L'État en Afrique. La politique du ventre, Paris, Fayard, 1989, p. 308.

27. Voir le site de Reporters sans frontières : www.rsf.org

28. R. de La Brosse, "Afrique subsaharienne en transition, des journaux nécessairement engagés ", Les cahiers du journalisme, n 6, octobre 1999, p. 102.

29. Le Reporter $\mathrm{n}^{\circ} 219$ du 13 juin 2003.

30. F. Ben Halla, « La démocratie par les médias », Géopolitique africaine, $\mathrm{n}^{\circ}$ 12, octobre 2003.

31. M.-S. Frère, Presse et démocratie en Afrique francophone. Les mots et les maux de la transition au Bénin et au Niger, Paris, Karthala, 2000, p. 257.

32. On appelle « reliance sociale » la fonction de la presse par laquelle le lecteur, en s'informant, s'intègre dans une communauté d'appartenance à la fois politique et sociale, se rapproche de ceux qui ont la même pratique médiatique, les mêmes centres d'intérêts, etc.

33. J. F. Bayart, op. cit., p. 223.

34. Le modèle français se caractérise notamment par l'importance du commentaire dans la retranscription des faits, les frontières perméables avec le monde politique, le fait de penser le journalisme comme une mission de service public avec rôle fort de l'État dans la structuration du champ. Lire E. Neveu, Sociologie du journalisme, Paris, La Découverte, 2001, pp. 12-18.

35. Le Reporter $n^{\circ} 219$ du 13 juin 2003.

36. L'Événement $\mathrm{n}^{\circ} 175$ du 25 juin 2003.

37. Tribune du peuple $\mathrm{n}^{\circ} 75 \mathrm{du} 18$ juin 2003.

38. On trouve de très nombreuses allégories dans le discours de Togo-Presse et de la presse de la mouvance présidentielle, qui placent Eyadéma sur le même plan qu'une divinité.

39. Tribune du peuple $\mathrm{n}^{\circ} 74 \mathrm{du} 11$ juin 2003.

40. La Dépêche $\mathrm{n}^{\circ} 215 \mathrm{du} 11$ juin 2003.

41. L'Événement $\mathrm{n}^{\circ} 174 \mathrm{du} 11$ juin 2003.

42. C.-M. Toulabor, «Jeux de mots, jeux de vilains. Lexique de la dérision politique au Togo », in J.-F. Bayart, A. Mbembe, C.-M. Toulabor, Le politique par le bas en Afrique noire. Contributions à une problématique de la démocratie, Paris, Karthala, 1992, p. 111.

43. Ibid., p. 68. 
44. Irrégularités sur les listes électorales et pressions sur les partis politiques d'opposition à la veille du vote ; irrégularités, omniprésence de l'armée, opacité et violences entraînant au moins deux morts le jour du scrutin.

45. Le Guide $\mathrm{n}^{\circ} 40 \mathrm{du} 11$ juin 2003.

46. Le régime RPT s'est beaucoup appuyé sur les chefs traditionnels, leaders d'opinion de leur communauté, pour son assise en milieu rural. Lire E. Adriaan, B. Van Roueroy Van Nieeuwaal, op. cit.

47. G. Balandier, Le détour. Pouvoir et modernité, Paris, Fayard, p. 41.

48. Togo-Presse $\mathrm{n}^{\circ} 6551$ du 20 juin 2003.

49. Politicos $\mathrm{n}^{\circ} 158 \mathrm{du} 27$ juin 2003.

50. G. Balandier, op. cit., p. 25.

51. Ibid., p. 93.

52. On peut répartir les 45 groupes ethniques en trois grands groupes : le groupe Éwé qui occupe tout le Sud du pays représente environ $45 \%$ de la population, le groupe des Kabyè-Tem, localisé au Nord, $25 \%$ et le groupe Paragouma $15 \%$. À côté de ces trois grands groupes, on dénombre quelques petits groupes minoritaires.

53. Voir Louise Bourgault, Mass Media in Subsaharan Africa, Bloomington IN, Indiana University Press, 1995, et M.-S. Frère, Presse et démocratie en Afrique francophone, op. cit.

54. F. Agblemagnon, Sociologie des sociétés orales d'Afrique Noire, Les Evés du Sud-Togo, Paris, Éditions Silex, 1984, p. 45.

55. Fils de Sylvanus Olympio et ennemi héréditaire d'Eyadéma, Gilchrist Olympio fait régulièrement les gros titres de la presse d'opposition, personnalisant plus que tout autre l'opposition au régime. Il jouit d'une grande notoriété auprès de la population. Depuis le retour du multipartisme, le pouvoir RPT fait tout pour l'écarter du pays et des compétitions électorales.

56. F. Agblemagnon, op. cit., p. 187.

57. M.-S. Frère, op. cit., p. 257.

58. Notons que, comme l'arbre à palabre, la presse représente un espace politique inégalitaire et sexué - les jeunes en sont écartés et les femmes restent moins alphabétisées et intéressées par le fait politique que les hommes.

59. Le « Petit» en éwé.

60. D.-C. Martin (dir.), Nouveaux langages du politique en Afrique orientale, Paris, Karthala/Kenya, IFRA, 1998, pp. 262-279.

\section{RÉSUMÉS}

Comment fonctionne la presse dans un Etat au pouvoir sans partage, le Togo, contraint in fine de se libéraliser quelque peu ? Presse célébrant le pouvoir du chef, ou presse d'opposition, le Président est partout, dans un média qui est à la fois acteur et témoin de la vie politique. Après un rapide historique de la presse togolaise, puis un état des lieux lors du tournant de 2003, l'article étudie son contenu, en dégageant ses deux rhétoriques dominantes - celle du pouvoir, qui instrumentalise un imaginaire local, et celle des gouvernés, empreinte de dérision. L'accent est mis enfin mis sur les trajectoires de réinterprétation de l'oralité qui se sont mises en place dans l'écrit journalistique. 
How the press is managed in an autocratic country - Togo - when is established power has to partially liberalise its policy? Be it the newspapers celebrating the national leader, or the opposition press, the President is everywhere, in a media which is both an actor and a witness of the political life. An overview of the historical background of the Togolese Press is followed by a review of the media and its structure at the turning point of 2003. The paper then analyses the content of the newspapers. It reveals a double rhetoric: the first from those in power that manipulates local imagination using universal propaganda techniques, and the second from the people, that uses derision. Finally, the research emphasizes the process of reinterpreting oral language that took place in journalistic writing.

\section{INDEX}

Keywords : printed media, state power, derision, orality, hybridisation

Mots-clés : presse, pouvoir d'état, dérision, oralité, hybridation

Index géographique : Togo

\section{AUTEUR}

\section{BRICE RAMBAUD}

Brice Rambaud a fait de nombreux stages de journalisme en Afrique subsaharienne avant de se consacrer à la recherche. Doctorant en sciences de l'information et de la communication, il consacre sa thèse à une étude comparative de la presse francophone et anglophone en Afrique, à travers la notion d'appropriation de modèles importés. Son travail porte plus spécifiquement sur le Burkina Faso et le Kenya, pour lequel il est allocataire ponctuel de l'Institut français de recherche en Afrique à Nairobi. Parmi ses publications : A. Lenoble-Bart et B. Rambaud, « Traditions libérales des médias. "Deux nations à la destinée commune" ", Politique Africaine, n 97, mars 2005, pp. 82-98.

brice.rambaud@laposte.net 